\title{
Role of MRI in Detection and Local Staging of Rectal Cancer
}

\author{
AHMED H. HASSAN BEK, M.Sc.; SHAYMAA H.M. SALAH, M.D. and MOHAMED A. ALY, M.D. \\ The Department of Radio-Diagnosis and Interventional Radiology, Faculty of Medicine, Ain Shams University
}

\begin{abstract}
Background: Colorectal cancer is one of the most frequent causes of cancer-related death worldwide. An accurate preoperative rectal cancer staging is crucial to the correct management of the disease. Despite great controversy around this issue, pelvic magnetic resonance is said to be the standard modality.

Aim of Study: The aim of this study is to assess the role of MRI in detection and local staging of rectal cancer.

Patients and Methods: 30 patients with rectal carcinoma were included with MRI and histopathological assessment of cancer staging to evaluate MRI accuracy. All patients received pre-operative neoadjuvant chemotherapy.

Results: T3 staging with MRI, calculation of sensitivity, specificity, negative and positive predictive value were $73.3 \%$, $40 \%, 60 \%$ and $55 \%$ respectively. T4 staging with MRI, calculation of sensitivity, specificity, negative and positive predictive value were $100 \%, 77 \%, 100 \%$ and $40 \%$ respectively.

Conclusion: MRI has a good sensitivity for tumor staging and nodal staging.
\end{abstract}

Key Words: Colorectal cancer $-M R I-T-N$ staging.

\section{Introduction}

COLORECTAL Cancer (CRC) is a heterogeneous disease that occurs in the colon and the rectum [1] The colon has 4 sections; the ascending, transverse, descending, and sigmoid colon, and the latter is where most CRC arise [2]. The majority of CRC develop slowly from adenomatous polyps or adenomas [3]. Recently, several studies suggested that CRC is a result of many factors, which are not only inherited but also acquired over the life course of the individual [4].

CRC incidence and mortality rates vary markedly around the world. Globally, CRC is the third most commonly diagnosed cancer in males and

Correspondence to: Dr. Ahmed H. Hassan Bek,

The Department of Radio-Diagnosis and Interventional Radiology, Faculty of Medicine, Ain Shams University the second in females according to the World Health Organization. Rates are substantially higher in males than in females [5]. The highest incidence rates are in Europe, and North America, and the lowest rates are found in Africa and South-Central Asia. These geographic differences appear to be due to differences in dietary and environmental exposures [6,7].

Low socioeconomic status is also associated with an increased risk for the development of CRC, potentially modifiable life style factors such as physical inactivity, unhealthy diet, smoking, and obesity are thought to account for a major proportion (estimates of one-third to one-half) of the socioeconomic role in development of CRC $[8,9]$.

Magnetic Resonance Imaging (MRI) is a powerful imaging technique for producing accurate anatomical images [10]. As compared to other crosssectional imaging modalities, MRI provides superior soft-tissue contrast and has no ionizing radiation exposure $[11,12]$. MRI scanners use strong magnetic fields, magnetic field gradients, and radio waves to generate images of the organs in the body. Magnetic resonance imaging is a medical application of Nuclear Magnetic Resonance (NMR) [13,14]

\section{Patients and Methods}

This was a cross sectional study that included 30 patients diagnosed with rectal cancer. The patients were selected from those attending the Radio diagnosis and Intervention Department, Ain Shams University Hospital in the period between June 2019 and March 2020.

\section{Ethical considerations:}

The study was approved from the Ethical Committee of the Department of Radio Diagnosis and Intervention, Faculty of Medicine, Ain Shams 
University. An informed consent was obtained from each patient.

\section{Inclusion criteria:}

1- Patients over 18 years old.

2- Patients with biopsy-proven rectal cancer.

\section{Exclusion criteria:}

A- Presence of general contraindications for MRI:

While in-situ metal implants are considered as absolute contraindication for MRI, there are other situations that possess relative contraindication for MRI.

\section{I- Absolute contraindications for MRI [15]:}

1- Pacemaker, defibrillator or wires other than sternal wires.

2- Metallic foreign body in the eye-these might move or heat during scanning resulting in serious eye injury.

3- Deep brain stimulator.

4- Bullets or gunshot pellets-near great vessels or vital organs.

5- Magnetic dental implants.

6- Drug infusion devices.

II- Relative contraindications for MRI [15]:

1- Other types of implants; surgical clips, wire sutures, screws or mesh, ocular prosthesis, penile prosthesis, joint replacement or prosthesis.

2- Morbidly obese patients might find it difficult to fit into the bore of the MRI.

3- Claustrophobic patients (might require sedation).

4- Surgery in the previous 6 weeks.

5- Significant pain might limit a patient's ability to lie still.

\section{B- Presence for contraindications for administra- tion of contrast media:}

In patients with poor renal function, there is a risk of Nephrogenic Systemic Fibrosis (NSF) associated with gadolinium chelate injections. Patients with known, or at risk of, renal impairment need to have their renal function assessed before MRI in order to determine whether administration of gadolinium contrast is safe or not [3].

Other acute reactions to gadolinium may be classified into major or severe and minor, and subdivided into local and general. The total incidence of adverse reactions to MRI contrast agents ranges approximately between $2 \%$ and $4 \%$. Most frequently, minor, general reactions are nauseas, emesis, hives, headache, while local reactions are: Skin irritation, itching and coolness. Cases of major acute adverse reactions to gadolinium, such as laryngospasm and anaphylaxis rarely occur [3] .

\section{Study procedure:}

1- Full history taking.

2- Full clinical examination to exclude any contraindication to MRI.

3- Magnetic resonance imaging:

- Pelvic MRI was performed on a $1.5 \mathrm{~T}$ magnet (Philips Acheiva, Guildford Business Park, Guildford, Surrey, Netherlands) with pelvic phased array coil and rectal gel administration.

- Only 2D T2-weighted (T2W) sequences were recommended for both primary and restaging.

- All patients underwent imaging while in the prone position following the placement of a small Foley catheter in the rectum and insufflation of approximately 200 to $300 \mathrm{~cm}^{3}$ of room air. No bowel preparation was used. A sagittal fast-spoiled gradient echo sequence was used to localize the lesion. This was followed by axial, conventional, spin echo T2-weighted images.

- Coronal and sagittal fast, spin echo T2weighted images was obtained. All images were interpreted by the same radiologist. Specific comment was made regarding depth of invasion of the rectal wall, adjacent organ involvement, and the presence of lymphadenopathy.

\section{MRI interpretation criteria:}

\section{A- T staging interpretations [16]:}

- $\mathrm{T} 1$ was staged if the tumor be confined to the mucosal layer of the rectal wall.

- T2 was staged if there is invasion of the rectal layer up to the muscularis propria, with no penetration of the muscularis propria or perirectal fat.

- T3 was staged if there is invasion of all rectal layers with perirectal fat infiltration yet without pelvic organ involvement.

- T4 was staged if there is invasion of mesorectal fascia and visceral peritoneum or surrounding organ infiltration.

\section{B- Lymph node staging interpretations [16]:}

- N0 was diagnosed if there is no lymph node metastasis. 
- N1 was diagnosed if there is metastasis in one to three lymph nodes.

- N2 was diagnosed if there is metastasis in four or more perirectal lymph nodes.

\section{C- Main outcome measures:}

Calculation of sensitivity, specificity, and accuracy for invasion through the bowel wall and lymph node status.

\section{Surgical and pathological plan-up:}

All cases were operated upon usually after 1 month from the last MRI and the post-operative specimens were compared with pre-operative MRI results.

All patients underwent anterior perineal resection, low anterior resection, or pelvic exenteration according to the location, and extension of previously diagnosed rectal cancer.

Post-operative pathological staging was correlated with the pre-operative MRI findings.

\section{Data management and analysis:}

The collected data was revised, coded, tabulated and introduced to a computer using Statistical package for Social Science (IBM Corp. Released 2011. IBM SPSS Statistics for Windows, Version 20.0. Armonk, NY: IBM Corp). Data was presented and suitable analysis was done according to the type of data obtained for each parameter.

\section{Results}

\section{I- Epidemiological data:}

The current study include 30 patients diagnosed with colorectal cancer. The patients were $18(60 \%)$ males and $12(40 \%)$ females. The patients' minimum age was 21 years and maximum was 75 years with mean of $48 \pm 14$ years old.

\section{II- Clinical characteristics of patients' malignan- cies:}

Location of the rectal cancer in the included patients was as follows: $3(10 \%)$ in upper rectum, $7(23 \%)$ in middle rectum and $20(67 \%)$ in lower rectum.

Regarding histopathological type, 20 (66.7\%) patients suffered from adenocarcinoma and 10 (33.3\%) patient suffered from mucinous adenocarcinoma Fig. (1).

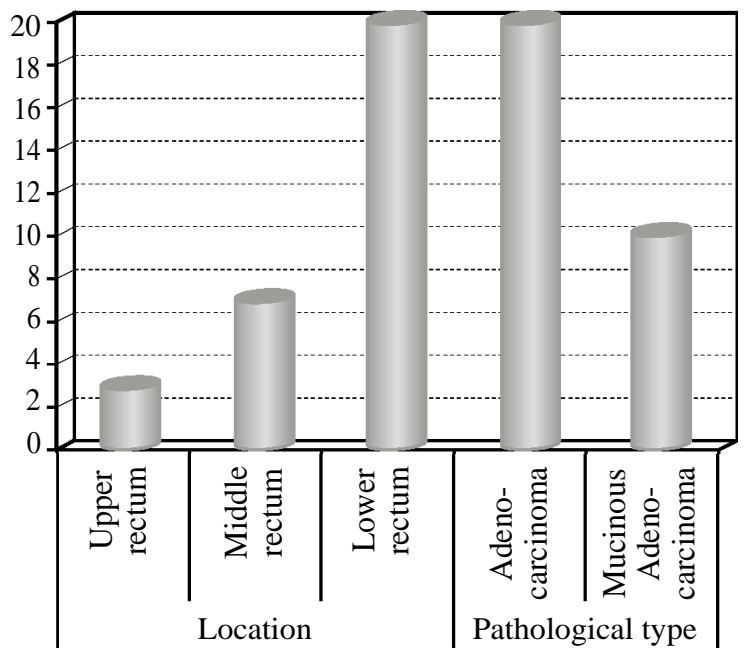

Fig. (1): Clinical characteristics of patients' malignancies.

\section{III- MRI staging of included patients:}

MRI T staging of the included patients revealed that $20(66.7 \%)$ patients had T3 stage and 10 $(33.3 \%)$ patients had T4 stage.

While, MRI N staging revealed that $2(6.7 \%)$ patients had N0, $8(26.7 \%)$ patients had N1 and $20(66.6 \%)$ patients had N2 stage Fig. (2).

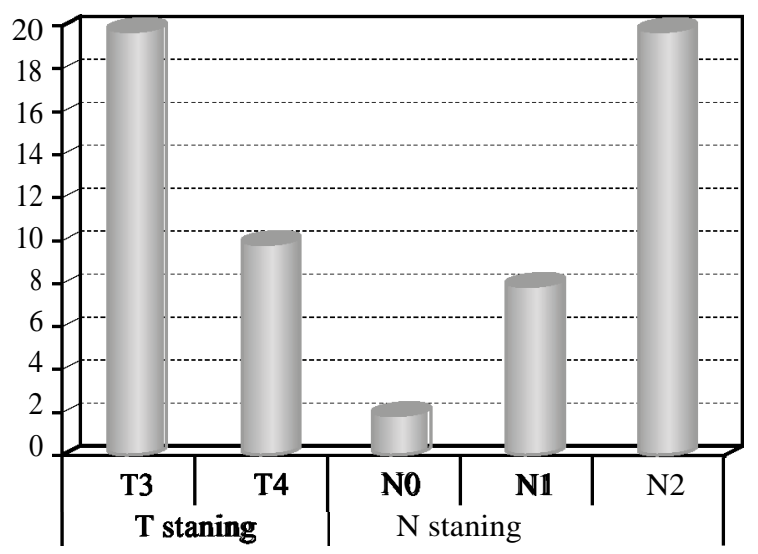

Fig. (2): MRI staging of included patients.

\section{IV-Pathological staging of included patients:}

Pathological T staging of the included patients revealed that $3(10 \%)$ patients had T0 stage, 1 (3.3\%) patient had T1 stage, $7(23.3 \%)$ patients had T2 stage, $16(53.3 \%)$ patients had T3 stage and $4(13.3 \%)$ patients had T4 stage.

While, pathological $\mathrm{N}$ staging revealed that 22 $(73.3 \%)$ patients had N0, $5(16.7 \%)$ patients had N1 and $3(10 \%)$ patients had N2 stage Figs. $(3,4)$. 


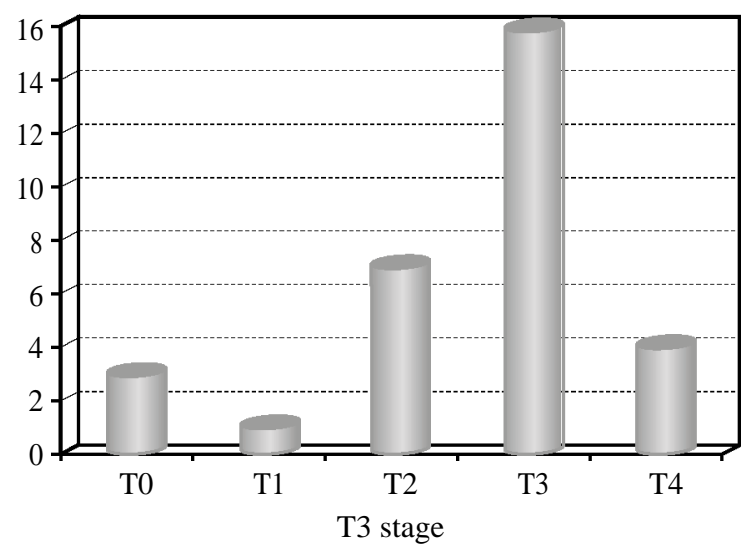

Fig. (3): Pathological staging of included patients (T staging).

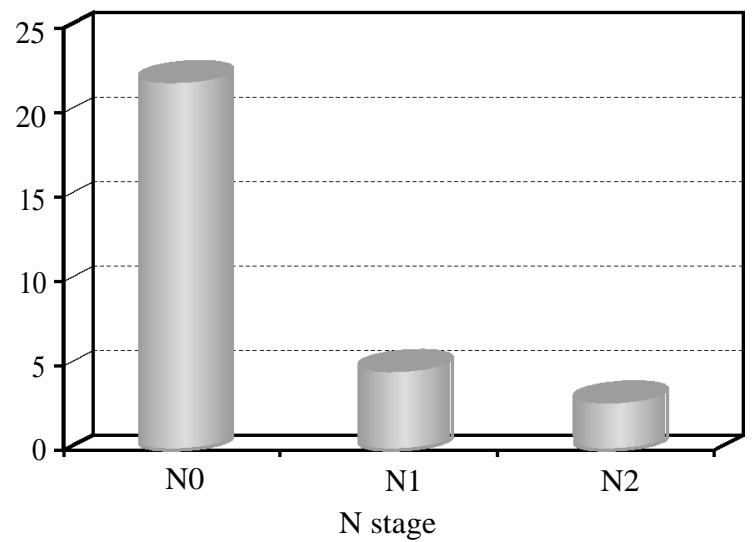

Fig. (4): Pathological staging of included patients ( $\mathrm{N}$ staging).

\section{$\mathrm{V}$ - Assessment of MRI accuracy in relevance to pathological examination:}

A- Assessment of MRI T staging accuracy:

MRI diagnosed 20 patients with T3 stage with only 11 cases were proven by histopathological assessment (agreement 55\%). Also, MRI diagnosed 10 patients with $\mathrm{T} 4$ stage with only 4 cases proven by histopathological assessment (agreement 40\%).

Correlation of T staging between MRI and histopathology was statistically significant ( $p$ value $=0.000$ ) reflecting major downstaging of $\mathrm{T}$ stage after receiving chemotherapy Fig. (5).

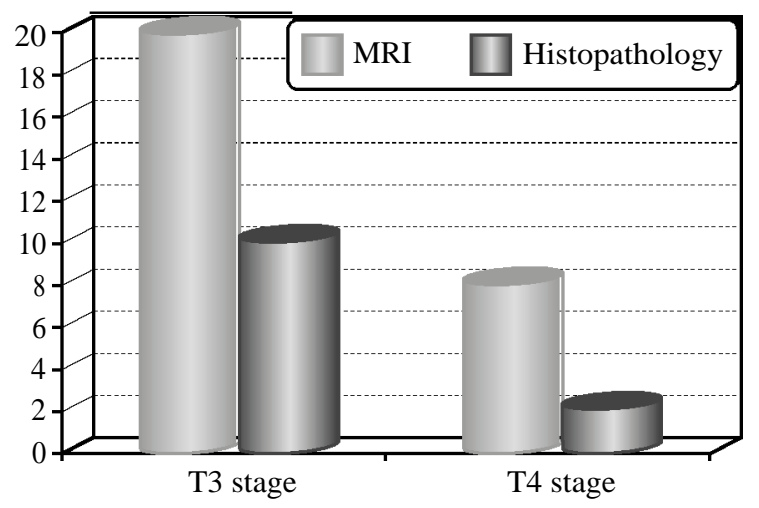

Fig. (5): MRI T stage accuracy assessment.
Regarding accuracy of diagnosis with MRI, of the 20 patients diagnosed with T3 stage 11 patients were correctly stated while, 9 patients were overstated.

On the other hand, the 10 patients diagnosed with T4 stage, only 4 were correctly stated while 6 were overstated (Table 1).

Table (1): Overview of accuracy of MRI diagnosis regarding $\mathrm{T}$ staging.

\begin{tabular}{lccc}
\hline Stage & Correctly stated & Overstated & Understated \\
\hline T3 & $11(55 \%)$ & $9(45 \%)$ & 0 \\
T4 & $4(40 \%)$ & $6(60 \%)$ & 0 \\
\hline
\end{tabular}

Regarding T3 staging with MRI, calculation of sensitivity, specificity, Negative and positive predictive value (NPV and PPV) were 100\%, 69\%, $100 \%$ and $55 \%$ respectively.

On the other hand, T4 staging with MRI, calculation of sensitivity, specificity, negative and positive predictive value (NPV and PPV) were $100 \%, 77 \%, 100 \%$ and $40 \%$ respectively (Table 2) \& Figs. $(6,7)$.

Table (2): Sensitivity, specificity, NPV and PPV for MRI T staging.

\begin{tabular}{lccll}
\hline & Sensitivity & Specificity & NPV & PPV \\
\hline T3 staging & $73.3 \%$ & $40 \%$ & $60 \%$ & $55 \%$ \\
T4 staging & $100 \%$ & $77 \%$ & $100 \%$ & $40 \%$ \\
\hline
\end{tabular}

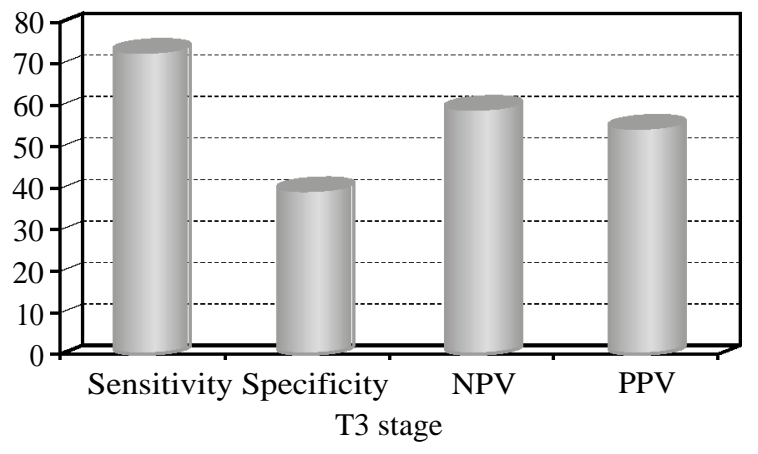

Fig. (6): Sensitivity, specificity, NPV and PPV for MRI T3 staging.

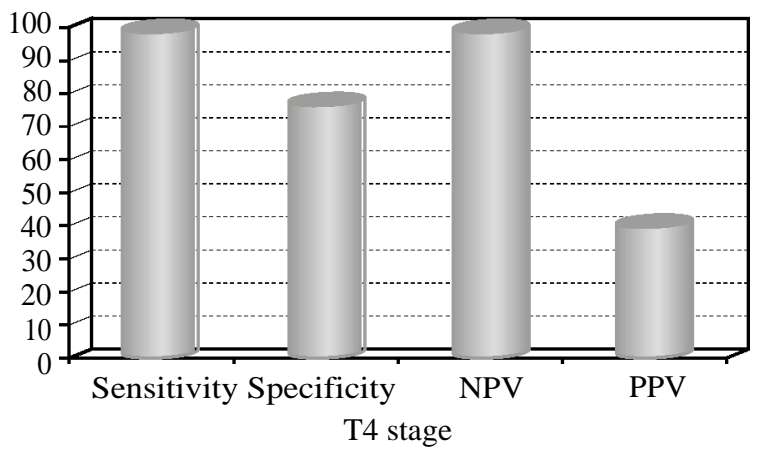

Fig. (7): Sensitivity, specificity, NPV and PPV for MRI T4 staging. 


\section{B- Assessment of MRI N staging accuracy:}

MRI diagnosed 2 patients with N0 stage which were proven by histopathological assessment (agreement 100\%), 8 patients with N1 stage with only 2 cases proven by histopathological assessment (agreement 25\%) and 20 patients with N2 stage with only 3 cases proven by histopathological assessment (agreement 15\%).

Correlation of $\mathrm{N}$ staging between MRI and histopathology was statistically significant ( $p$ value $=0.000$ ) reflecting major downstaging of $\mathrm{T}$ stage after receiving chemotherapy Fig. (8).

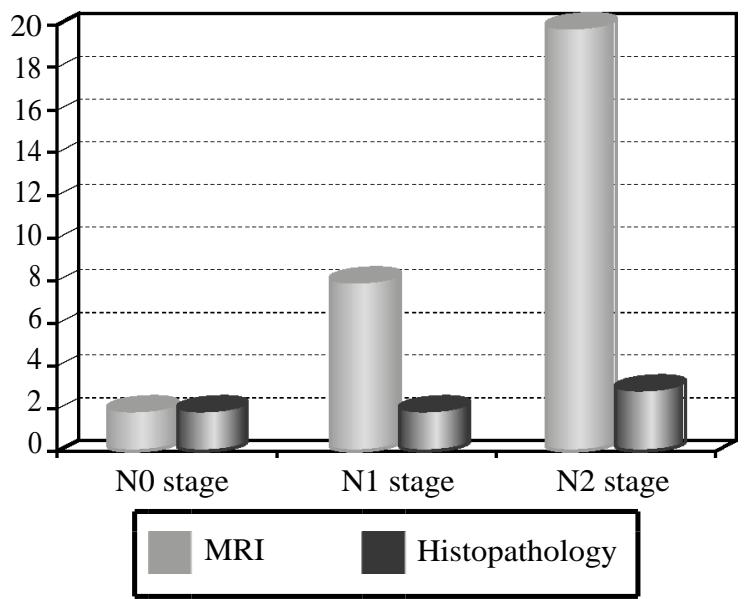

Fig. (8): MRI N stage accuracy assessment.

Regarding accuracy of diagnosis with MRI, of the 2 patients diagnosed with NO stage they were correctly stated.

On the other hand, the 8 patients diagnosed with N1 stage, only 2 were correctly stated while 6 were overstated. Also, the 20 patients diagnosed with N2 stage were only 3 correctly stated while 17 were overstated (Table 3 ).

Table (3): Overview of accuracy of MRI diagnosis regarding $\mathrm{N}$ staging.

\begin{tabular}{lclc}
\hline Stage & Correctly stated & Overstated & Understated \\
\hline N0 & $2(100 \%)$ & 0 & 0 \\
N1 & $2(25 \%)$ & $6(75 \%)$ & 0 \\
N2 & $3(15 \%)$ & $17(85 \%)$ & 0 \\
\hline
\end{tabular}

Regarding N0 staging with MRI, calculation of sensitivity, specificity, NPV and PPV were $10 \%$, $100 \%, 28.6 \%$ and $100 \%$ respectively. N1 staging with MRI, calculation of sensitivity, specificity, NPV and PPV were 40\%, 76\%, $86 \%$ and $25 \%$ respectively. Finally, N2 staging with MRI, calculation of sensitivity, specificity, NPV and PPV were $100 \%, 37 \%, 100 \%$ and $15 \%$ respectively (Table 4) \& Figs. $(9,10,11)$.
Table (4): Sensitivity, specificity, NPV and PPV for MRI N staging.

\begin{tabular}{lccll}
\hline & Sensitivity & Specificity & NPV & PPV \\
\hline N0 staging & $10 \%$ & $100 \%$ & $28.6 \%$ & $100 \%$ \\
N1 staging & $40 \%$ & $76 \%$ & $86 \%$ & $25 \%$ \\
N2 staging & $100 \%$ & $37 \%$ & $100 \%$ & $15 \%$ \\
\hline
\end{tabular}

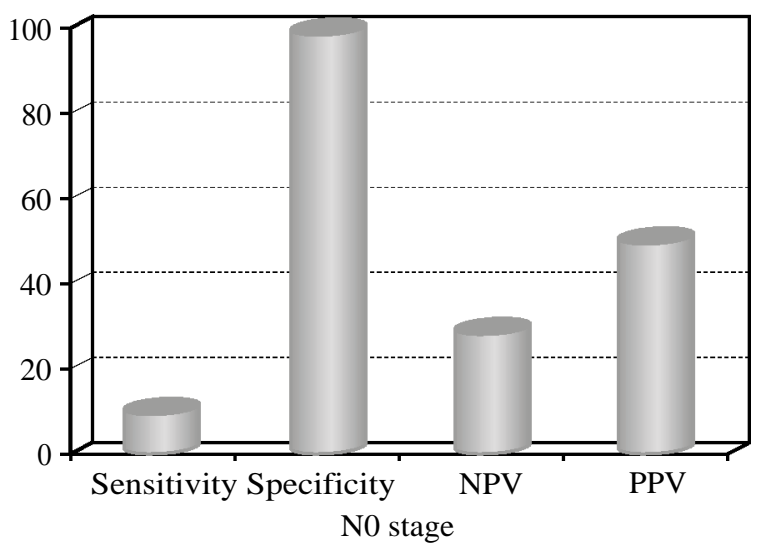

Fig. (9): Sensitivity, specificity, NPV and PPV for MRI NO staging.

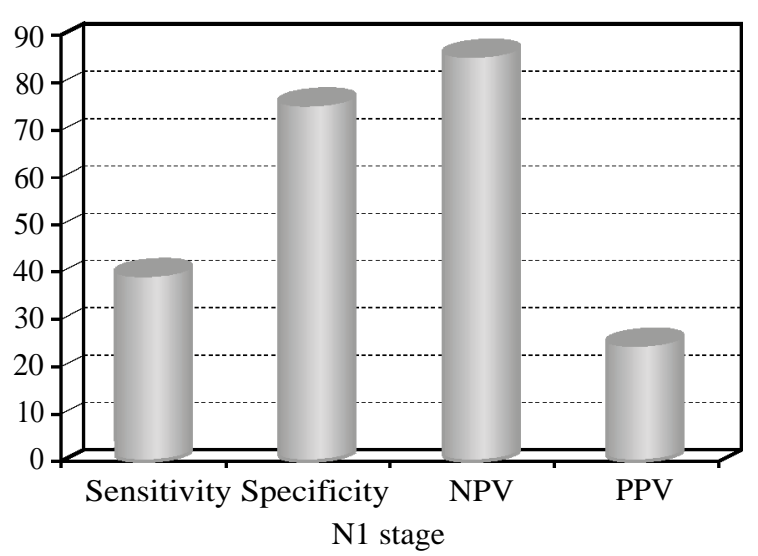

Fig. (10): Sensitivity, specificity, NPV and PPV for MRI N1 staging.

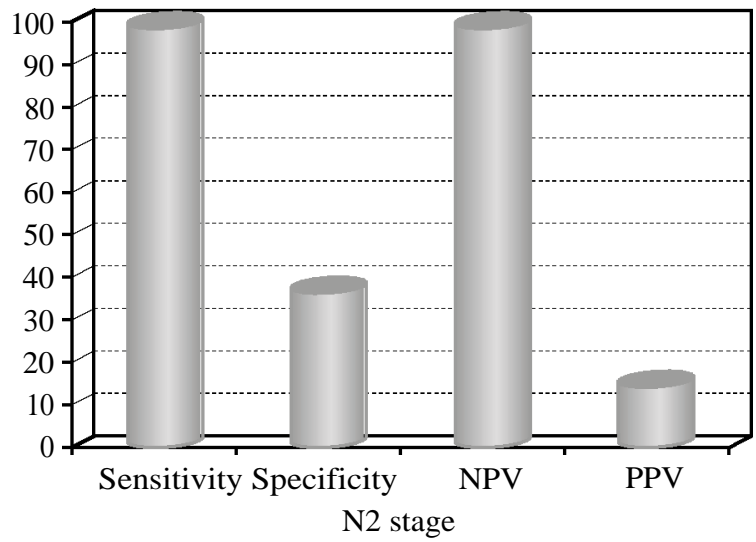

Fig. (11): Sensitivity, specificity, NPV and PPV for MRI N2 staging. 


\section{Discussion}

This study aimed to assess the role of MRI in detection and local staging of rectal cancer. We included 30 patients with biopsy-proven rectal cancer and comparison between pretreatment MRI and post-operative histopathological assessment was done.

Regarding MRI T staging, MRI diagnosed 20 patients with T3 stage with only 11 cases were proven by histopathological assessment (agreement 55\%). Also, MRI diagnosed 10 patients with T4 stage with only 4 cases proven by histopathological assessment (agreement 40\%). Moreover, accuracy of diagnosis with MRI was; of the 20 patients diagnosed with $\mathrm{T} 3$ stage 11 patients were correctly stated while, 9 patients were overstated. On the other hand, the 10 patients diagnosed with $\mathrm{T} 4$ stage, only 4 were correctly stated while 6 were overstated. T3 staging sensitivity, specificity, NPV and PPV were $73.3 \%, 40 \%, 60 \%$ and $55 \%$ respectively while, T4 staging sensitivity, specificity, NPV and PPV were 100\%, 77\%, 100\% and 40\% respectively.

MRI N staging results were a bit different, MRI diagnosed 2 patients with N0 stage which were proven by histopathological assessment (agreement $100 \%$ ), 8 patients with N1 stage with only 2 cases proven by histopathological assessment (agreement $25 \%$ ) and 20 patients with N2 stage with only 3 cases proven by histopathological assessment (agreement 15\%). Regarding accuracy of diagnosis with MRI, of the 2 patients diagnosed with NO stage they were correctly stated. On the other hand, the 8 patients diagnosed with N1 stage, only 2 were correctly stated while 6 were overstated. Also, the 20 patients diagnosed with N2 stage were only 3 correctly stated while 17 were overstated. NO staging sensitivity, specificity, NPV and PPV were $10 \%, 100 \%, 28.6 \%$ and $100 \%$ respectively. N1 staging sensitivity, specificity, NPV and PPV were $40 \%, 76 \%, 86 \%$ and $25 \%$ respectively and finally, N2 staging sensitivity, specificity, NPV and PPV were $100 \%, 37 \%, 100 \%$ and $15 \%$ respectively.

Liu et al., (2019) studied diagnostic performance of MRI and CT for local staging of sigmoid and descending colon cancer. They included 116 patients with sigmoid or descending colon cancer who underwent both MRI and CT before surgery, MRI and CT images were separately reviewed by two independent and blinded radiologists to assess the following features: T-stage, and lymph node metastases $(\mathrm{N}+)$. MRI achieved correct T-stage in
81 of 116 patients $(69.8 \%)$, sensitivity was $89.2 \%$ and specificity was $81.8 \%$. The sensitivity and specificity for identifying nodal involvement were $70.9 \%$ and $72.6 \%$ respectively [17]

Abreu and Martins, (2015) evaluated magnetic resonance accuracy in preoperative rectal cancer staging comparing with the anatomo-pathological results. They included 41 patients with rectal carcinoma. The respective sensitivity, specificity, positive and negative predictive values were $33.3 \%$, $94.7 \%, 33.3 \%$ and $94.7 \%$ for $\mathrm{T} 1 ; 62.5 \%, 32 \%$, $37.0 \%$ and $57.1 \%$ for $\mathrm{T} 2 ; 31.8 \%, 79 \%, 63.6 \%$ and $50 \%$ for $\mathrm{T} 3$ and $27.8 \%, 87 \%, 62.5 \%$ and $60.6 \%$ for $\mathrm{N}$ [1].

Van den Broek et al., (2017) study the accuracy of MRI in restaging locally advanced rectal cancer after pre-operative chemoradiation. They included 48 patients with locally advanced rectal cancer, $T$ stage was correctly predicted in $47 \%$ to $68 \%$ and $\mathrm{N}$ stage in $68 \%$ to $70 \%$. Overstaging was more common than understaging. Positive Predictive Values (PPV) among the 3 readers for T0 were $0 \%$, and Negative Predictive Values (NPVs) varied from $84 \%$ to $85 \%$. For T1/2, PPVs and NPVs were $50 \%$ to $67 \%$ and $72 \%$ to $90 \%$, and for T3/4 they were $54 \%$ to $62 \%$ and $33 \%$ to $78 \%$. PPVs and NPVs for N0 stage were $81 \%$ to $95 \%$ and $58 \%$ to 73\% [18]

Another study was done by Nerad et al., (2017) 55 patients with biopsy-proven colorectal carcinoma. MRI had a high sensitivity $(72 \%-91 \%)$ and specificity $(84 \%-89 \%)$ in detecting T3/T4 tumors (35/55) and a low sensitivity (43\%-67\%) and high specificity (75\%-88\%) in detecting T3cd/T4 tumors (15/55). MRI also had moderate sensitivity and specificity in the detection of nodal involvement [19].

\section{Limitations:}

There are several limitations in the present study, first small number of patients included in this study interfered with accuracy of results concluded. Second, absence of T1/T2 stages was beyond our capabilities in recruiting included patients, all patients included were T3/T4 stages. Absence of post chemotherapy MRI data hindered our analysis to overview the exact response of the patients to the neoadjuvant chemotherapy. Also, absence of data regarding mesorectal fat involvement (MRF), Circumferential Resection Margin (CRM) and Extramural Vascular Invasion (EMVI) was due to technical difficulties in collecting the data of the patients. 


\section{Conclusion:}

- CRC diagnosis is accurately diagnosed using MRI.

- MRI proves to be sensitive in $\mathrm{T}$ staging diagnosis but specificity still to be under further assessment.

- MRI has poor sensitivity in determining $\mathrm{N}$ staging in CRC.

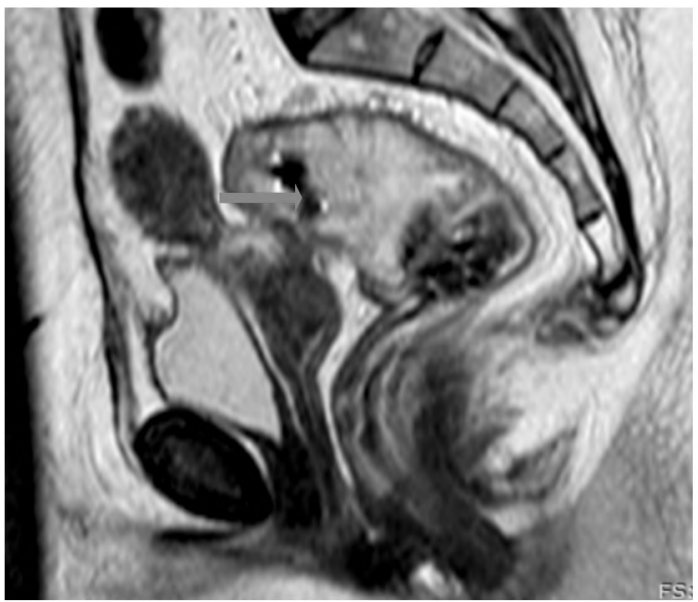

(A)

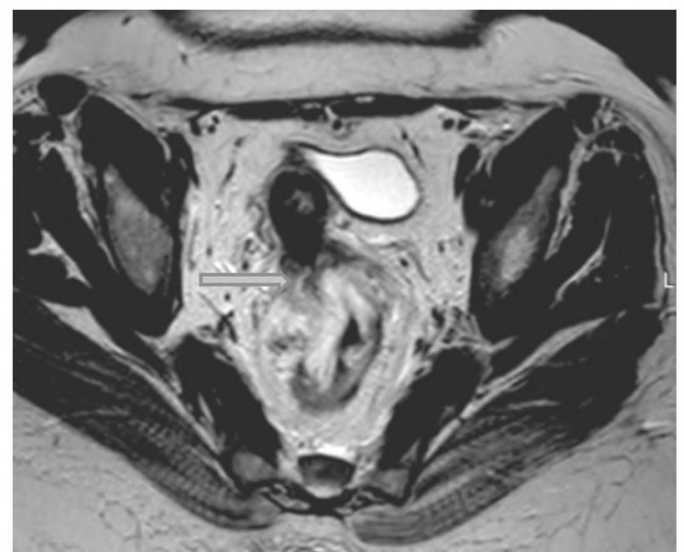

(C)
- Most rectal cancer patients showed downstaging of their malignancies after neoadjuvant therapy.

\section{Case presentation:}

Case (1): Female patient 43 years old complain from constipation clinical rectal examination and endoscopy was done reveled hard mass $9 \mathrm{~cm}$ from anal verge biopsy was taking reveled mucinous adenocarcinoma, MRI was done.

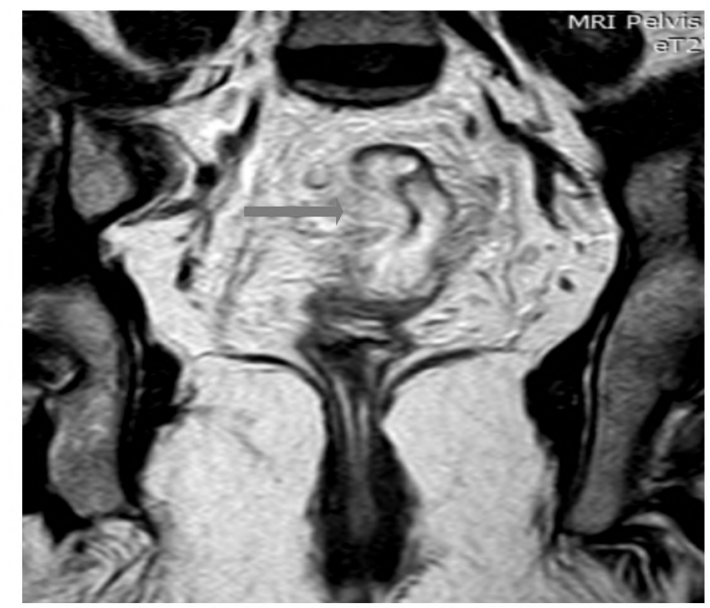

(B)

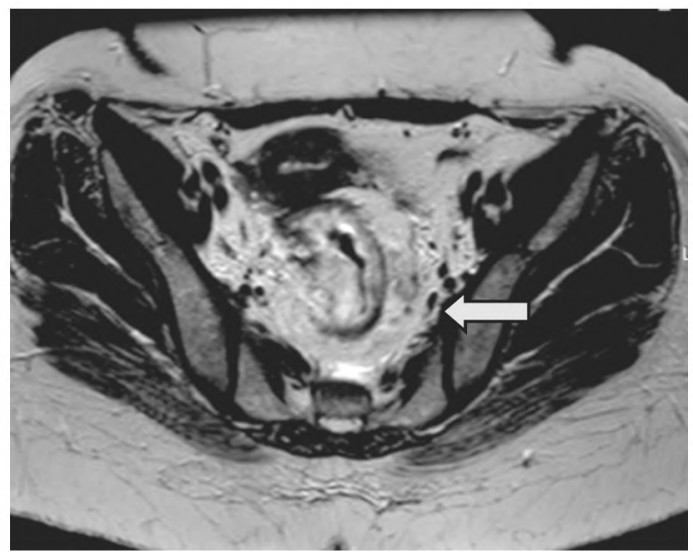

(D)

Case (1): (A \& B) Sagittal and coronal imaging show irregular circumferential wall thickening is seen in mid rectum extended to sigmoid colon $9 \mathrm{~cm}$ from anal verge (red arrow), (C) Axial T2WI shows serosial infiltration and infiltrate to the posterior wall of the lower part of cervix (blue arrow), (D) Axial T2WI shows per rectal round soft tissue mass represented lymph node deposit (green row).

MRI diagnosis: Picture suggestive of rectal carcinoma with extension classification as T4N1.

The patient underwent abdominoperonial resection after neoadjuvant therapy, pathology of the excised specimen reveled muscines adenocarcinoma with positive lymph node metastasis ( 2 out of 3 node) with pathological staging (T4N1).

Case (2):

Male patient 52 years anal discharge, clinical rectal examination and endoscopy was done re- vealed hard mass $3 \mathrm{~cm}$ from anal verge, biopsy reveled moderate differentiated adenocarcinoma, MRI examination was done. 


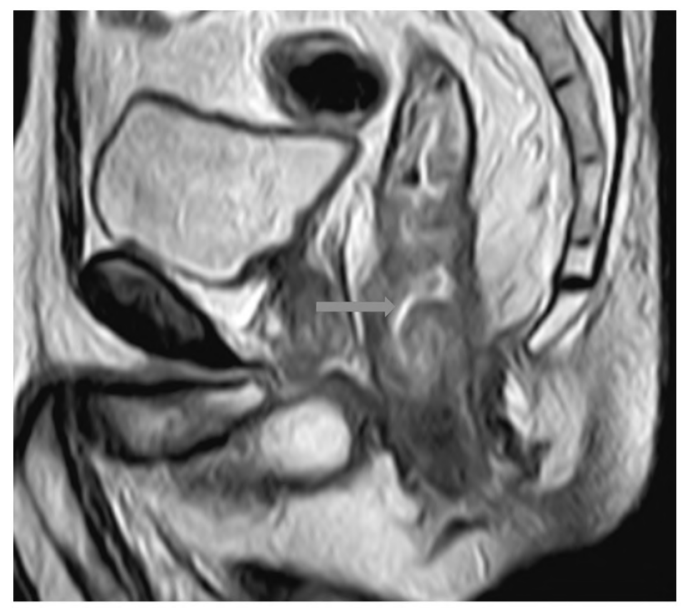

(A)

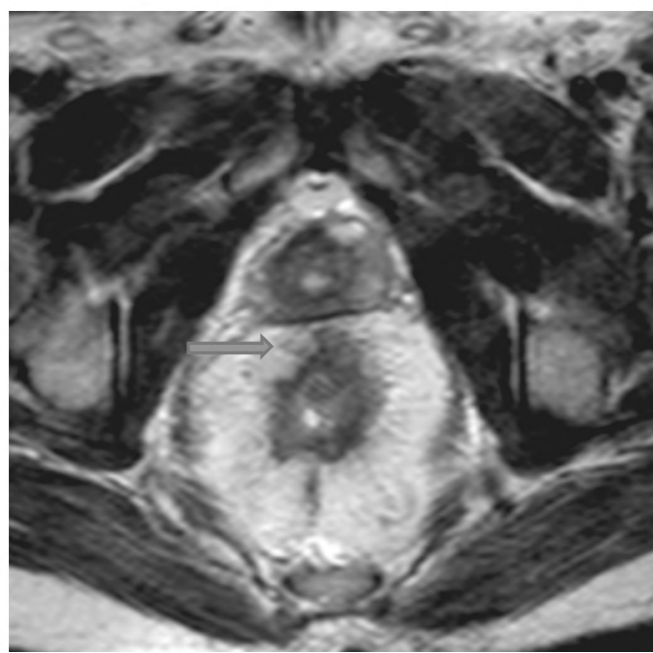

(C)

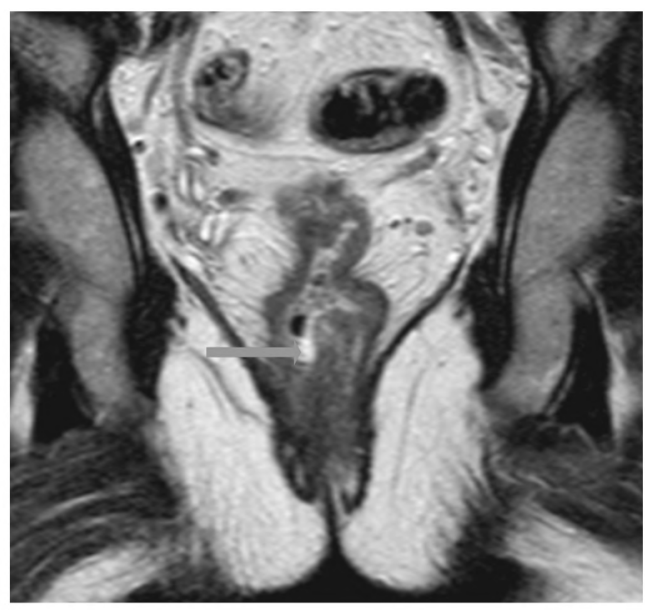

(B)

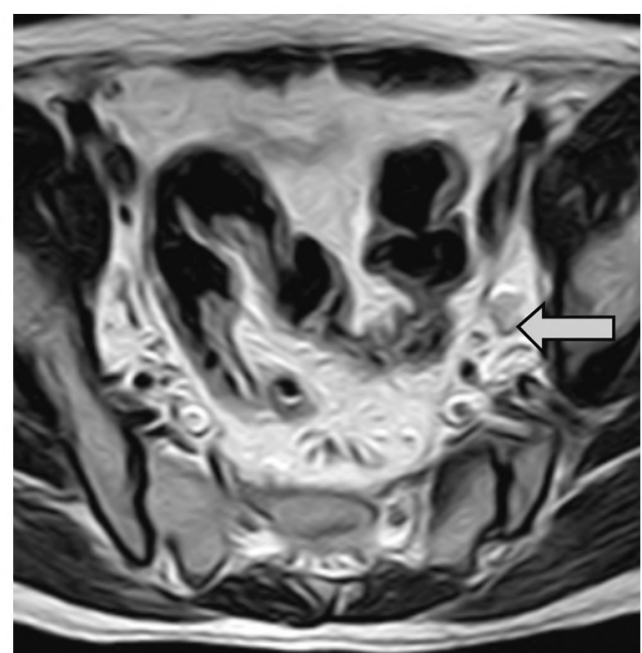

(D)

Case (2): (A \&B) Sagittal and coronal imaging shows show fungation mass in lower rectum in poterolateral wall with diffuse circumferential wall thickening in mid and lower rectum $3.4 \mathrm{~cm}$ from anal verge (red arrow), (C) Axial T2WI shows mesorectal fat involvement (blue arrow), (D) Axial T2WI show left internal iliac lymph node (green row).

MRI diagnosis: Diffuse mid and lower rectal wall thickening with fungation mass poterolateral and perirectal fat invasive classification as T3N2.

The patient underwent abdominoperonial resection after neoadjuvant therapy, pathology of the excised specimen reveled moderate differentiated adenocarcinoma with negative lymph node metastasis (0 out of 10) with pathological staging (T2N0).

\section{References}

1- ABREU S.F. and MARTINS S.F.: Pre-operative staging of rectal cancer with MRI: Correlation with pathologic staging. Journal of Coloproctology (Rio de Janeiro), Jun., 35 (2): 77-82, 2015.

2- ARAN V., VICTORINO A.P., THULER L.C., et al.: Colorectal cancer: Epidemiology, disease mechanisms and interventions to reduce onset and mortality. Clinical colorectal cancer, Sep. 1, 15 (3): 195-203, 2016.

3- BOHORQUEZ M., SAHASRABUDHE R., CRIOLLO A., et al.: Clinical manifestations of colorectal cancer patients from a large multicenter study in Colombia. Medicine, Oct., 95 (40), 2016.

4- LEVINE J.S. and AHNEN D.J.: Adenomatous polyps of the colon. New England Journal of Medicine, Dec., 14, 355 (24): 2551-7, 2006.
5- SCHOEN R.E., PINSKY P.F., WEISSFELD J.L., et al.: Colorectal-cancer incidence and mortality with screening flexible sigmoidoscopy. New England Journal of Medicine, Jun. 21, 366 (25): 2345-57, 2012.

6- DOUBENI C.A., MAJOR J.M., LAIYEMO A.O., et al.: Contribution of behavioral risk factors and obesity to socioeconomic differences in colorectal cancer incidence. Journal of the National Cancer Institute, Sep. 19, 104 (18): 1353-62, 2012.

7- FITZMAURICE C., AKINYEMIJU T.F., AL LAMI F.H., et al.: Global, regional, and national cancer incidence, mortality, years of life lost, years lived with disability, and disability-adjusted life-years for 29 cancer groups, 1990 to 2016: A systematic analysis for the global burden of disease study. JAMA oncology, Nov. 1, 4 (11): 1553 68, 2018.

8- LAUENSTEIN T., RAMIREZ-GARRIDO F., KIM Y.H., et al.: Nephrogenic systemic fibrosis risk after liver 
magnetic resonance imaging with gadoxetate disodium in patients with moderate to severe renal impairment: Results of a prospective, open-label, multicenter study. Investigative radiology, Dec., 50 (6): 416, 2015.

9- PARRY S., WIN A.K., PARRY B., et al.: Metachronous colorectal cancer risk for mismatch repair gene mutation carriers: The advantage of more extensive colon surgery Gut., Jul. 1, 60 (7): 950-7, 2011.

10- PARK S.Y., CHO S.H., LEE M.A., et al.: Diagnostic performance of MRI-versus MDCT-categorized T3cd/T4 for identifying high-risk stage II or stage III colon cancers: A pilot study. Abdominal Radiology, May 15, 44 (5): 1675-85, 2019.

11- TAYLOR F. G., QUIRKE P., HEALD R.J., et al.: Preoperative magnetic resonance imaging assessment of circumferential resection margin predicts disease-free survival and local recurrence: 5-year follow-up results of the MERCURY study. Journal of Clinical Oncology, Jan. 1; 32 (1): 34-43, 2014

12- SCHMOLL H.J., VAN CUTSEM E., STEIN A.E., et al.: ESMO Consensus Guidelines for management of patients with colon and rectal cancer. A personalized approach to clinical decision making. Annals of oncology, Oct. 1; 23 (10): 2479-516, 2012.

13- MORRIS S.A. and SLESNICK T.C.: Magnetic resonance imaging. Visual Guide to Neonatal Cardiology. Apr. 16: 104-8, 2018.
14- PATEL U.B., BLOMQVIST L.K., TAYLOR F., et al.: MRI after treatment of locally advanced rectal cancer: How to report tumor response-the MERCURY experience. American Journal of Roentgenology, Oct., 199 (4): W48695, 2012.

15- CARAIANI C., PETRESC B., DONG Y. and DIETRICH C.F.: Contraindications and adverse effects in abdominal imaging. Medical ultrasonography, Dec. 9, 21 (4): 45663, 2019.

16- ARYA S., DAS D., ENGINEER R. and SAKLANI A.: Imaging in rectal cancer with emphasis on local staging with MRI. The Indian journal of radiology \& imaging, Apr., 25 (2): 148, 2015.

17- LIU L.H., LV H., WANG Z.C., et al.: Performance comparison between MRI and CT for local staging of sigmoid and descending colon cancer. European journal of radiology, Dec. 1, 121: 108741, 2019.

18- VAN DEN BROEK J.J., VAN DER WOLF F.S., LAHAYE M.J., et al.: Accuracy of MRI in restaging locally advanced rectal cancer after preoperative chemoradiation. Diseases of the Colon \& Rectum. Mar. 1, 60 (3): 274-83, 2017.

19- NERAD E., LAMBREGTS D.M., KERSTEN E.L., et al.: MRI for local staging of colon cancer: Can MRI become the optimal staging modality for patients with colon cancer? Diseases of the Colon \& Rectum, Apr. 1, 60 (4): 385-92, 2017.

\section{دور التصوير بالرنين المغناطيسى

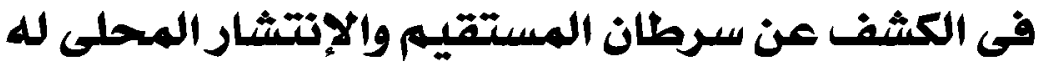

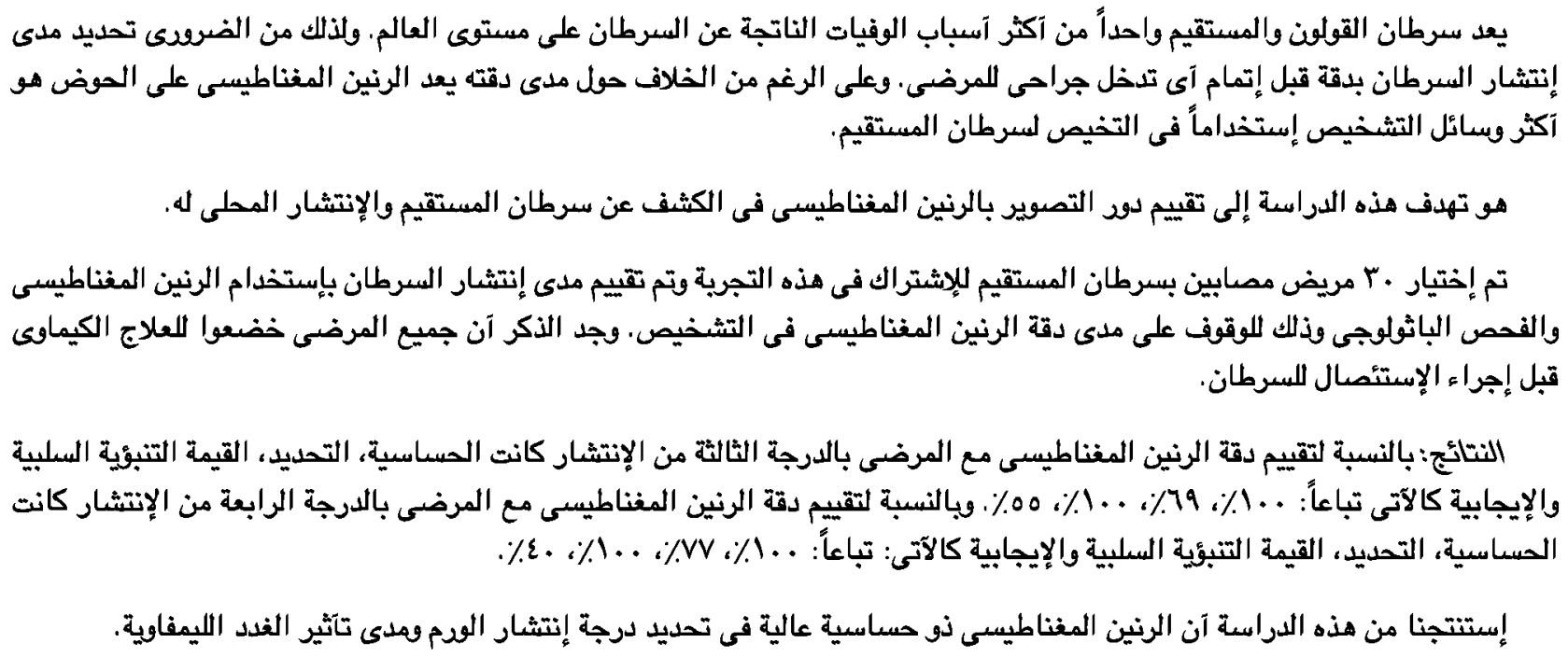

